\title{
A regional framework for action on substance use and public health
}

\author{
Khalid Saeed ${ }^{1}$ and Mahshid Taj ${ }^{2}$
}

Substance use is a public health problem worldwide. According to United Nations Office on Drugs and Crime (UNODC) estimates, there has been an increase of more than $10 \%$ over recent years in cannabis use in 9 countries in the Region; while the prevalence of opiate use in Afghanistan, the Islamic Republic of Iran and Pakistan is among the highest in the world. The estimated prevalence for drug dependence in the North Africa and Middle East region is $0.29 \%$, which is 1.3 times higher than the global prevalence (1). Emergencies and civil unrest are known as important factors in the exacerbation of the situation (2).

At an international level increasing attention is being paid to adopting a more public health-oriented approach. This was highlighted in the outcome document of the United Nations General Assembly Special Session on Drugs (UNGASS) in 2016, which called for a balanced approach between law enforcement and the health sector to counter the world drug problem and inclusion of substance use targets under the health goal of the 2015 Sustainable Development Goals (SDGs) agenda $(3,4)$.

The Eastern Mediterranean Region (EMR) faces particular challenges in implementing the operational recommendations of the UNGASS 2016 outcome document and reporting on the SDG targets. It comprises 22 economically diverse countries, with their own cultures and characteristics. A substantial number of these countries are experiencing insecurity, war and humanitarian crisis. In complex humanitarian situations, there is attrition in the capacity of the health and social systems to respond to the increased needs and demands. Since different countries in the Region are at varying stages of development, including the development of their mental health systems, WHO Regional office for the Eastern Mediterranean (EMRO) has devised a classification of countries into three groups that are similar in terms of their population health outcomes, health system performance and level of health expenditure. This facilitates a more meaningful comparison between countries, and for recommendations appropriate to countries' current levels of health system and socio-economic development (5).

The Substance Use Atlas 2015 is designed to collect information and report on the agreed upon indicators and targets (6). The current iteration of the Atlas includes contributions from all countries of the Region and provides data on the status of substance use services and will serve as a benchmark to monitor progress. The key findings from the Substance Use Atlas as pertains to EMR countries indicate that (5):

- The estimated prevalence of opioid use varies greatly, but some countries have the highest rates in the world.

- More than $60 \%$ of the countries have a legislative provision for offering voluntary treatment for drug use disorders as an alternative or in addition to criminal sanctions.
- Government financing is the most common financing method for treatment services for substance use disorders.

- $60 \%$ of countries have specialized treatment facilities for substance use disorders, but the regional median number of beds reserved for the treatment of substance use disorders is 0.7 per 100000 population.

- Cannabis and opioids are the main substances reported as the primary drugs at treatment entry; pharmacotherapies for opioid dependence are available in less than $25 \%$ of countries; needle/syringe exchange programmes are available in one-third of the countries.

- Most countries have not implemented screening and brief interventions for substance use in primary health care.

- More than half of countries do not have an epidemiological data collection system for substance use; $45 \%$ of countries do not have a system of data collection based on health services delivery for substance use.

In order to implement the operational recommendations of the outcome document of the UNGASS 2016 Health Action Plan within the Region, EMRO has developed a framework to operationalise the proposed actions by converting them into practical and concrete recommendations for intervention. The principles guiding the development of the proposed Regional Framework are evidence-informed, specific, relevant, feasible and internally consistent. It also 
incorporates a set of SMART indicators that will be used to monitor and evaluate progress.

In order to inform and support the Framework for Implementation, EMRO commissioned teams of international experts to develop evidence briefs on key issues in strengthening the public health response to drug use problems. The remit of these briefs is to review and summarize national and international evidence relevant to countries in the Region, and suggest strategic interventions for ministries of health that are affordable, cost effective and feasible to strengthen the public health response to the substance use problem in countries of the Region. These briefs, which are brought together in this theme issue along with the Framework for Implementation, represent the current best evidence for strengthening the public health response to the drug use problem.
The key recommendations include:

- Integration of screening and brief interventions for substance use problems in primary health care interventions packages.

- Ensuring availability of essential medicines for management of substance use disorders while strengthening regulatory systems to minimize misuse of prescription medicines.

- Building up the capacity of the workforce in the health and social welfare sectors to provide evidence-informed care for substance use disorders.

- Allocating a specific budget within the health and welfare sector to address substance use disorders.

- Revising policies, plans and legislations conforming to international standards and conventions.

- Assessing national resource needs and corresponding prioritization of budgetary allocations; taking care to protect people from potentially catastrophic costs of treatment.

- Implementing specified "best buy" interventions for promotion and prevention programmes.

- Routine collection and reporting on resources, service availability, and coverage for substance use disorders, and developing systems for monitoring drug-related deaths and communicable diseases prevalence associated with substance use.

- Enhancing capacities to carry out priority research to inform policy and service development.

WHO will continue to provide systematic support to the Member States in order to strengthen the public health response to the drug use problem. It will be coordinating our support with relevant national and international organizations and entities to achieve the targets set out in the 2015 Sustainable Development Agenda.

\section{References}

1. United Nations Office on Drugs and Crime (UNODC). World Drug Report 2016. New York: United Nations; 2016 (https://www.unodc.org/doc/wdr2016/WORLD_DRUG_REPORT_2016_web.pdf, accessed 8 March 2017).

2. Hanna FB. Alcohol and substance use in humanitarian and post-conflict situations. East Mediterr Health J. 2017; 23(3): 233-37.

3. United Nations Office on Drugs and Crime (UNODC). Outcome document of the 2016 United Nations General Assembly Special Session on the world drug problem. New York: United Nations; 19-21 April 2016 (https://www.unodc.org/documents/postungass2016//outcome/V1603301-E.pdf, accessed 8 March 2017).

4. United Nations General Assembly. Transforming our world: the 2030 agenda for sustainable development (70/1). New
York: United Nations; 2015 (http://www.un.org/ga/search/ view_doc.asp?symbol=A/RES/70/1, accessed 8 March 2017).

5. WHO Regional Office for the Eastern Mediterranean. Health systems strengthening in countries of the Eastern Mediterranean Region: challenges, priorities and options for future action (EM/RC59/Tech.Disc.1). Cairo: 59th Session Regional Committee for the Eastern Mediterranean; 2012 (http://applications.emro.who.int/docs/RC_Resolutions_2012_3_14693_ EN.pdf?ua=1, accessed 12 March 2017).

6. WHO Regional Office for the Eastern Mediterrnean. Atlas: substance use in the Eastern Mediterranean Region 2015. Cairo: Regional Offie for the Eastern Mediterranean; 2017 (http://apps.who.int/iris/bitstream/10665/254675/1/emropub_2017_19560.pdf, accessed 8 March 2017). 